\title{
Interleukine 4
}

L'interleukine 4 (IL-4) est une glycoprotéine sécrétée par un nombre restreint de cellules dont les lymphocytes $T$ et les basophiles/mastocytes. Elle agit en se fixant sur un récepteur de haute affinité présent en faible nombre sur de très nombreux types cellulaires comme les lymphocytes B et $T$, les granulocytes basophiles, éosinophiles et neutrophiles, les monocytes/macrophages, les fibroblastes et les cellules endothéliales. Les effets biologiques de l'IL-4 dépendent de l'état d'activation de la cellule cible, de l'environnement cellulaire et des autres cytokines. De manière indirecte, l'IL-4 peut moduler la production de cytokines des lymphocytes $\mathrm{T}$ et $B$, des macrophages et des fibroblastes. Elle joue un rôle majeur dans l'induction de la production d'IgE en induisant la commutation isotypique des cellules $B$ vierges, phénomène bloqué par les interférons. L'IL-4 pourrait ainsi, a priori, s'avérer utile comme agent antitumoral et anti-inflammatoire.

\section{Jacques Banchereau}

\section{ADRESSE}

J. Banchereau : directeur $d u$ laboratoire de recherches immunologiques. Schering-Plough, 27, chemin des Peupliers, 69570 Dardilly,

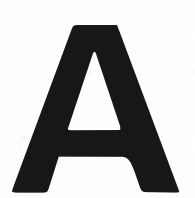

u cours des deux dernières décennies, il a été établi que les cellules du système immunitaire communiquent entre elles et avec les cellules des autres systèmes par des polypeptides appelés cytokines. Parmi celles-ci, l'interleukine 4 (IL-4) murine a été identifiée, en 1982, pour sa capacité à induire la prolifération de lymphocytes $B$ costimulés avec des anti-IgM et la sécrétion d'IgG $\mathrm{G}_{1}$ par les cellules $B$ activées avec le LPS (lipopolysaccharide). Cette protéine a d'abord été appelée BCGF I ( $B$ cell growth factor I) puis BSF 1 ( $B$ cell stimulatory factor 1) et finalement IL-4 quand un ADN complémentaire spécifique a été isolé en 1986 par deux équipes $[1,2]$. Cette même année, un ADNc spécifique de l'IL-4 humaine a été isolé [3]. Finalement, en 1989 et 1990, des ADNc spécifiques des récepteurs de l'IL-4 murins et humains ont été isolés [4-7]. Nous résumerons les principales propriétés de l'IL-4 et de son récepteur et spéculerons au sujet des perspectives thérapeutiques ouvertes par l'identification de cette molécule. Comme plusieurs centaines de références sur l'IL-4 ont été publiées au cours de ces trois dernières années, les lecteurs intéressés par une bibliographie détaillée peuvent se référer aux références les plus récentes [4-7] et aux revues récentes [8-11]. 


\begin{tabular}{|c|c|c|}
\hline \multicolumn{3}{|c|}{ PROPRIÉTÉS DE L'INTERLEUKINE 4} \\
\hline & Homme & Souris \\
\hline $\begin{array}{l}\text { Protéine précurseur (1) } \\
\text { Protéine sécrétée(1) } \\
\text { Sites de N-glycosylation } \\
\text { Taille du gène (2) } \\
\text { Introns du gène } \\
\text { Localisation chromosomique du gène } \\
\text { Sources cellulaires } \\
\text { Récepteur (Kd) }\end{array}$ & $\begin{array}{c}153 \\
129 \\
2 \\
10 \\
3 \\
5 q 23-31 \\
\text { Cellules } \mathrm{T} \\
\\
\approx 10-10 \mathrm{M}\end{array}$ & $\begin{array}{c}140 \\
120 \\
3 \\
6,7 \\
3 \\
11 \\
\text { Cellules T } \\
\text { Cellules stromales } \\
\text { de la moelle osseuse } \\
\text { Mastocytes } \\
\approx 10-10 \mathrm{M}\end{array}$ \\
\hline
\end{tabular}

(1) Acides aminés; (2) en kilobases; Kd : constante de dissociation.
CD45RA + (lymphocytes "naïs ") et les cellules T CD8 + ne semblent pas capables d'en produire.

Les cellules de type $\mathrm{TH}_{1}$ favorisent les réponses d'hypersensibilité de type retardé, à médiation cellulaire, alors que les cellules de type $\mathrm{TH}_{2}$ favorisent les réponses anticorps.

Les mécanismes contrôlant la différenciation des lymphocytes $\mathrm{T}$ naïfs en cellules $\mathrm{TH}_{1}$ ou $\mathrm{TH}_{2}$ restent à élucider, mais dépendent en partie de l'antigène puisque les allergènes et certains antigènes parasitaires favorisent l'apparition de cellules de type $\mathrm{TH}_{2}$. Le complexe majeur d'histocompatibilité est aussi impliqué, puisque les souris $\mathrm{Balb} / \mathrm{c}$, très sensibles à la leishmaniose, sont incapables de monter une réponse de type $\mathrm{TH}_{1}$ spécifique des leishmanies, alors que ce sont les cellules $\mathrm{TH}_{1}$ qui conferent la résistance à la leishmaniose des souris de type C57BI/6. Les hormones corticostéroïdes peuvent, in vitro et in vivo, favoriser la production d'IL-4 par les lymphocytes $\mathrm{T}$ et inhiber la production d'IL-2.

Il est intéressant de noter que les cellules $\mathrm{TH}_{1}$ et $\mathrm{TH}_{2}$ s'inhibent mutuellement. Ainsi l'IFN $\gamma$ (produit par les cellules $\mathrm{TH}_{1}$ ) bloque la prolifération des clones $\mathrm{TH}_{2}$ et la prolifération GM-CSF (5q23-31), le CSF-1 ou MCSF (5q33-1) et le récepteur du CSF-1 (5q31-32) (figure 1). Cette association génique se retrouve sur le chromosome $11 \mathrm{chez}$ la souris.

A l'opposé de l'IL-1 ou de l'IL-6, mais comme l'IL-2 ou l'interféron $\gamma$ $(\mathrm{IFN} \gamma)$, l'interleukine 4 est sécrétée par un nombre restreint de cellules et plus particulièrement par les lymphocytes $T$. Les études sur les clones de lymphocytes T CD4+ murins, ont montré une subdivision en deux sous-types : $\mathrm{TH}_{1}$ sécrétant IL-2 et IFN $\gamma$ et $\mathrm{TH}_{2}$ sécrétant IL-4 et IL-5 [12] (Tableau II, p. 948). Cette subdivision semble refléter un état final de maturation des lymphocytes CD4, consécutif à une stimulation antigénique répétée. En effet, il semble qu'un lymphocyte CD4 " naïf " produise essentiellement de l'IL-2 puis, en se différenciant, IL-2, IL-4, IL- 5 et IFN $\gamma$ et finalement soit IL-4 et IL-5 soit IL-2 et IFN $\gamma$. L'utilisation d'anticorps monoclonaux a permis d'identifier la présence de ces différentes popula-

tions de cellules CD4 dans la rate de souris. La situation semble plus complexe chez l'homme, où seulement une minorité de clones $\mathrm{T}$ possède les caractéristiques $\mathrm{TH}_{1}$ ou $\mathrm{TH}_{2}$ alors que la plupart sécrètent l'ensemble des cytokines. Cependant, les lymphocytes $\mathrm{T}$ de phénotype CD4 + CD45RA- (lymphocytes $T$ auxiliaires mémoires) produisent de l'IL-4 alors que les cellules CD4 +

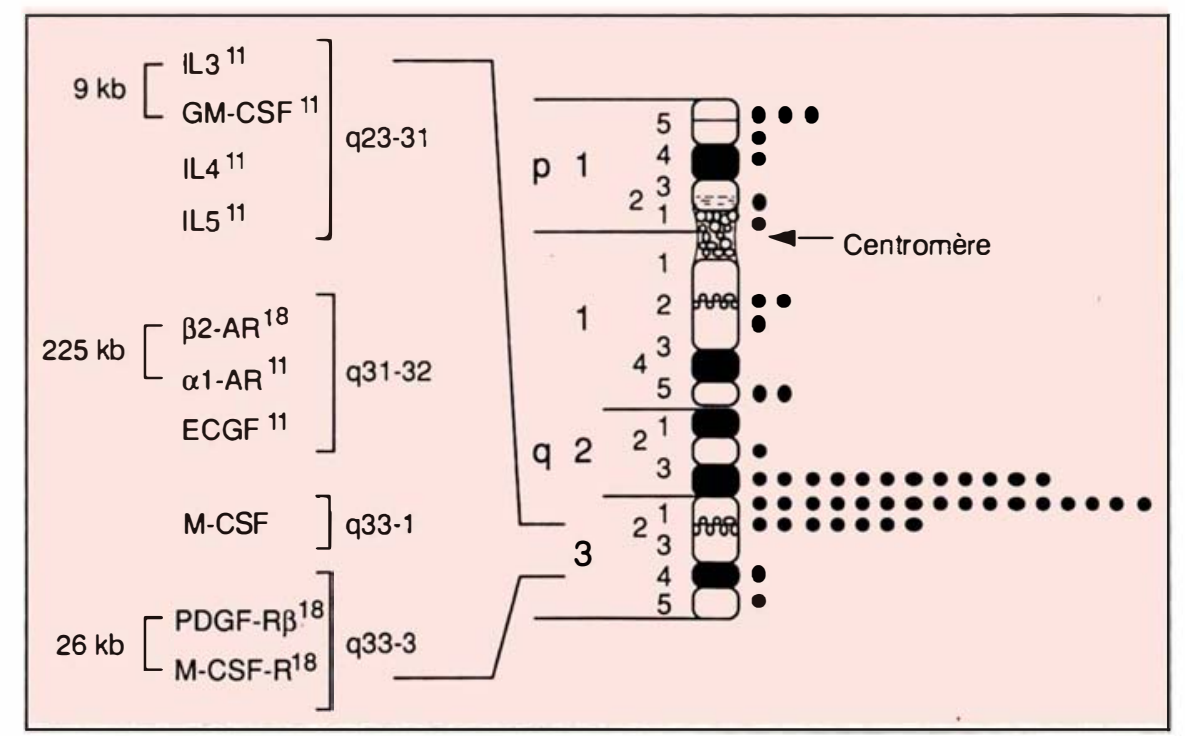

Figure 1. Gènes de cytokines et de leurs récepteurs sur le chromosome 5 humain. Les valeurs en kb représentent la distance séparant les gènes considérés. Les chiffres en index représentent la localisation chromosomique chez la souris. - : histogrammes des signaux d'hybridation obtenus avec une sonde $d^{\prime} I L-4$. 


\section{* GLOSSAIRE*}

IL-3, IL-4, IL-5 : interleukines 3, $4,5$.

GM-CSF : facteur de croissance des granulocytes et monocytes.

$\alpha 1 A R$ : récepteur $\alpha 1$ adrénergique.

$\beta 2 A R$ : récepteur $\beta 2$ adrénergique.

$\boldsymbol{E C G F}$ : endothelial cell growth factor.

PDGF-R : récepteur du facteur de croissance dérivé des plaquettes.

M-CSFR : récepteur du M-CSF (ou proto-oncogène c-fms).

M-CSF : facteur de croissance des macrophages.

LPS : lipopolysaccharides.

TNP : groupe hapténique.

$T N F$ : tumor necrosis factor.

$P G E$ : prostaglandine $E$.

IFN : interféron.

EPO : érythropoiétine.

IL-3R : récepteur de l'IL-3.

$I L-4 R$ : récepteur de l'IL-4.

G-CSFR : récepteur du G-CSF.

$I L-6 R$ : récepteur de l'IL-6.

$I L-2 R \beta$ : récepteur de l'IL-2 chaine $\beta$.

GM-CSFR : récepteur du GMSF.

\section{RÉFÉRENCES}

1. Lee F, Yokota T, Otsuka T, et al. Isolation and characterization of a mouse interleukin cDNA clone that expresses B cell stimulatory factor 1 activities and $T$ cell - and mast-cell - stimulating activities. Proc Nall Acad Sci USA 1986 ; 83 : 2061-5.

2. Noma $Y$, Sideras $T$, Naito $T$, et al. Cloning of cDNA encoding the murine IgG1 induction factor by a novel strategy using SP6 promotor. Nalure 1986 ; 319: 640-6.

3. Yokota T, Otsuka T, Mosmann T, et al. Isolation and characterization of a human interleukin cDNA clone, homologous to mouse B-cell stimulatory factor 1, that expresses B-cell-stimulatory activities. Proc Nall Acad Sci USA 1986 ; 83 : 5894-8.

4. Mosley B, Beckmann MP, March CJ, et al. The murine interleukin 4 receptor : molecular cloning characterization of secreted and membrane bound forms. Cell 1989 ; 59 : $335-48$.

5. Harada N, Castle BE, Gorman DM, et al. Expression cloning of a cDNA encoding the murine interleukin 4 receptor based on ligand binding. Proc Nall Acad Sci USA $1990 ; 87$ : 857-61. des clones $\mathrm{TH}_{1}$ est inhibée par un produit non caractérisé des cellules $\mathrm{TH}_{2}$. De plus, les clones $\mathrm{TH}_{2}$ sécrètent une cytokine (CSIF pour cytokine synthesis inhibitory factor) qui inhibe les fonctions des cellules $\mathrm{TH}_{1}$ [13] dont la production d'IFN $\gamma$. De plus, chez l'homme mais pas chez les souris, l'IL-4 produite par les cellules $\mathrm{TH}_{2}$ est aussi capable de bloquer la production d'IFN $\gamma$.

Chez la souris, d'autres cellules que les lymphocytes $\mathrm{T}$ peuvent sécréter de l'IL-4. En particulier l'IL-4 sécrétée par les mastocytes jouerait un rôle dans la prolifération autocrine de ces cellules en réponse au pontage des $\operatorname{IgE}$ de surface par un allergène. Il a aussi été montré que les cellules stromales de la moelle osseuse sécrètent de l'IL-4, qui aurait alors un rôle dans la prolifération et la différenciation des précurseurs hématopoïétiques des lymphocytes $B$.

\section{Interleukine-4 et lymphocytes B}

Les études in vitro ont démontré que l'IL-4 agit à de nombreuses étapes du développement (indépendantes de l'antigène) et de la différenciation (dépendante de l'antigène) des lymphocytes B (figure 2).

- Ontogénie et activation des lymphocytes B

Des expériences in vitro ont montré, chez la souris, que l'IL-4 stimule la prolifération des cellules pro-B. Il est possible que cet effet soit indirect car de telles cultures ne sont réalisables qu'en présence de cellules stromales qui pourraient, en réponse à l'IL-4, sécréter un facteur induisant la prolifération des cellules pro-B. L'IL-4 est aussi capable d'induire la maturation des cellules pro- $B$ en cellules pré-B (induction des chaînes $\mu$ intracytoplasmiques) et celle des cellules pré-B en cellules B immatures (induction des IgM de surface). Cependant, des signaux différents de l'IL-4 (autres cytokines et/ou contacts cellulaires) sont indispensables pour obtenir la maturation des progéniteurs B en cellules B matures. Sur les cellules B matures, l'IL-4 induit l'expression de l'antigène CD23, récepteur de faible affinité pour l'IgE, dont le rôle physiologique n'est pas clairement établi. L'IL-4 augmente l'expression des antigènes d'histocompatibilité de classe II à la surface des cellules B et, de cette façon, favoriserait la présentation de l'antigène par la cellule $B$ à la cellule T. Parallèlement, l'IL-4 facilite la formation de conjugués entre les cellules $\mathrm{T}$ et les cellules $\mathrm{B}$. Ces effets de l'IL-4 permettent donc une amplification de la réponse humorale.

- Prolifération des cellules B

L'IL-4 induit la prolifération de cel-
Tableau II

CYTOKINES PRODUITES PAR LES CELLULES DE SOURIS

\begin{tabular}{|l|c|c|c|c|}
\hline & CTL(1) & TH $_{\mathbf{1}}$ & TH $_{\mathbf{2}}$ & Mastocytes \\
\hline Interféron r & ++ & ++ & - & \pm \\
Interleukine 2 & \pm & ++ & - & \pm \\
Lymphotoxine & + & ++ & - & - \\
GM-CSF & ++ & ++ & + & $?$ \\
TNF (2) & + & ++ & + & - \\
Interleukine 3 & + & ++ & ++ & ++ \\
Met-enképhaline & + & + & ++ & $?$ \\
Interleukine 4 & - & - & ++ & ++ \\
Interleukine 5 & - & - & ++ & ++ \\
Interleukine 6 & - & - & ++ & ++ \\
CSIF lou IL-10 ?)(3) & $?$ & - & ++ & $?$ \\
\hline
\end{tabular}

(1) CTL : cellule T cytotoxique ; (2) TNF : facteur nécrosant des tumeurs ; (3) CSIF : facteur inhibant la synthèse des cytokines. ? : inconnu. (D'après Mosmann et Coffmann, 1989.) 


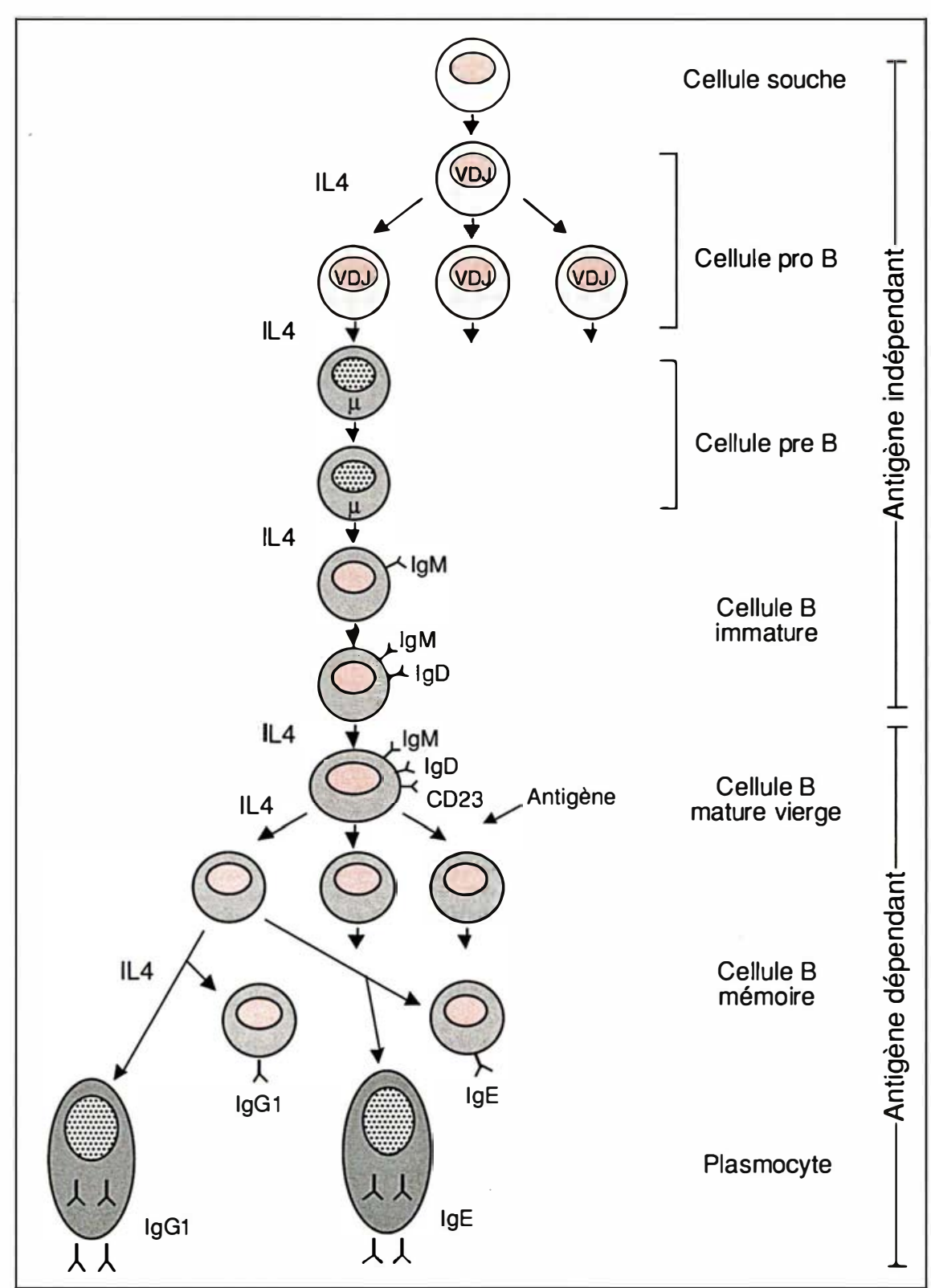

Figure 2. Sites d'action de I'IL-4 sur la lignée lymphocytaire B. VDJ : réarrangement des segments VDJ des gènes de chaînes lourdes d'immunoglobuline.

lules B costimulées avec des anticorps anti-IgM. Récemment, nous avons développé un nouveau système de prolifération des cellules B en pontant leur antigène $\mathrm{CD} 40$ grâce à un anticorps monoclonal anti-CD40 immobilisé. L'addition d'IL-4 à de telles cultures nous a permis d'établir des lignées continues de lymphocytes B strictement dépendantes de l'IL-4 et de l'anti-CD40. Ces lignées ne sont pas transformées par le virus $\mathrm{m} / \mathrm{s} n^{\circ} 10$, vol. 6, décembre 90
l'IL-4 bloque la prolifération induite par l'IL-2. Cet effet est particulièrement notable sur les cellules B leucémiques dont la prolifération induite par l'IL-2 est totalement inhibée par l'IL-4. Les mécanismes contrôlant ces effets activateurs et inhibiteurs de l'IL-4 sont mal connus, mais nos expériences récentes suggèrent que l'effet inhibiteur de l'IL-4 sur la prolifération dépendante de l'IL-2 pourrait être due à l'activation de l'adénylate cyclase.

- Différenciation des lymphocytes B

L'IL-4 modifie de façon importante la nature des isotypes sécrétés par les cellules B de souris activées par le LPS. En particulier, elle induit la production d' $\operatorname{IgG}_{1}$ et d'IgE par commutation au niveau des gènes des chaînes lourdes d'Ig. Chez l'homme, l'IL-4 induit la production d' $I g E$ par les cellules mononucléées totales ou par les cellules B purifiées infectées par le virus d'Epstein-Barr ou activées par des anticorps anti-CD40 immobilisés. Chez la souris et chez l'homme, la production d'IgE induite par l'IL-4 est fortement inhibée par les interférons $\alpha$ et $\gamma$. L'IL-4 stimule aussi la production d'IgM et d' $\operatorname{IgG}_{1}$ par les cellules $B$ humaines activées. De façon paradoxale, l'IL-4 inhibe la production d'IgG, IgA, IgM par les cellules mononucléées en réponse aux activateurs polyclonaux (nos résultats non publiés). La production d'anticorps spécifiques, en réponse aux conjugués trinitrophenyl (TNP) et au virus influenza, est aussi bloquée par l'IL-4. Ces effets inhibiteurs seraient liés à la production de facteurs suppresseurs par les monocytes.

Le rôle fondamental de l'IL-4 dans la production d'IgE in vivo a pu être démontré chez la souris [14]. En effet, l'administration d'anticorps neutralisant l'IL-4 bloque l'augmentation du taux d'IgE circulante induite par administration, à des souris, de parasites nématodes ou d'anticorps anti-IgD. L'injection d'interféron $\alpha$ ou $\gamma$ à des souris ainsi traitées bloque aussi l'augmentation du taux d'IgE circulante. Des essais pilotes chez des patients souffrant de syndrome hyper-IgE ont montré que les interférons peuvent induire une diminution du taux d'IgE sérique. 


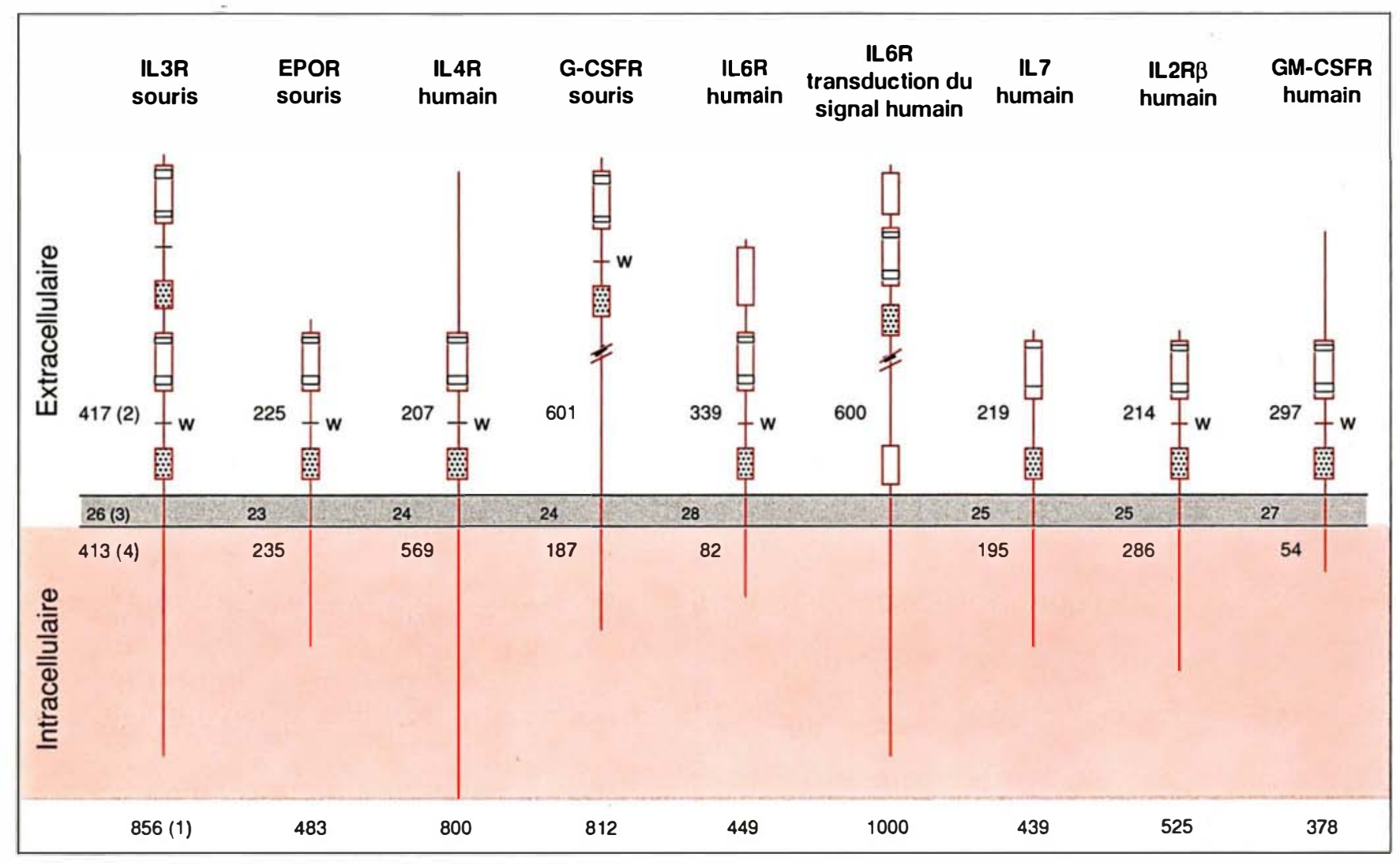

Figure 3. Le récepteur de I'IL-4 appartient à une nouvelle famille de récepteurs. Le premier motif conservé est le rectangle blanc avec des barres représentant les cystéines. Le rectangle hachuré représente le deuxième motif conservé. La structure immunoglobulinique du récepteur IL-6 est aussi représentée (rectangle blanc). W : tryptophane conservé. (1) : AA de la protéine mature; (2) : AA extracellulaires; (3): AA transmembranaires; (4): AA intracellulaires.

Toutefois, il reste à déterminer si ce traitement améliore l'état clinique de ces patients.

\section{Interleukine 4 et lymphocytes $T$}

L'IL-4, en costimulation avec un agent mitogène, induit la prolifération des thymocytes et des lymphocytes $\mathrm{T}$ matures. Elle entraîne aussi la maturation des thymocytes en induisant la disparition des cellules CD4 + CD8 + et l'apparition de cellules CD4 + $\mathrm{CD}^{-}{ }^{-}$et $\mathrm{CD} 8+\mathrm{CD}^{-}$. Paradoxalement, l'IL-4 est capable d'induire l'apparition de l'antigène CD8 sur les clones T CD4 + . Comme décrit précédemment pour les cellules $\mathrm{B}$, l'IL-4 bloque la prolifération des cellules $\mathrm{T}$ périphériques induite par l'IL-2. Toutefois l'IL-2 et l'IL-4 agissent en coo- de cellules $\mathrm{T}$ activées par leur récepteur à l'antigène.

L'IL-4 stimule le développement de cellules $\mathrm{T}$ cytotoxiques spécifiques de cellules stimulatrices allogéniques ou de cellules infectées par le virus influenza. En revanche, l'IL-4 bloque la production de cellules LAK (lymphokine activated killer) dépendante de l'IL-2.

\section{Interleukine 4 et cellules myélo-monocytaires}

Les études in vitro montrent que l'IL-4 peut inhiber ou stimuler la myélopoïèse. Elle inhibe l'apparition de colonies dépendantes du M-CSF et du GM-CSF, mais stimule la formation des colonies dépendantes du GCSF. En particulier, en combinaison avec l'IL-3, l'IL-4 joue un rôle important dans la genèse des granulocytes éosinophiles et basophiles.

L'IL-4 active les monocytes/macrophages en induisant un accroissement de taille, les antigènes d'histocompatibilité de classe II et l'antigène CD23. Cependant, elle inhibe l'expression des récepteurs pour les IgG (Fc $\gamma$ RI, Fc $\gamma$ RII, Fc $\gamma$ RIII) et, de ce fait, elle inhibe la cytotoxicité des macrophages dépendante d'anticorps. L'IL-4 bloque la production d'IL-1, IL-6, TNF $\alpha$ (tumor necrosis factor $\alpha$ ) et $\mathrm{PGE}_{2}$ (protaglandine $\mathrm{E}$ ) par les monocytes, mais stimule la production de G-CSF et M-CSF. Elle inhibe la production de superoxyde mais n'inhibe pas la phagocytose

L'IL-4 agit comme un stimulateur des granulocytes neutrophiles en accroissant la phagocytose et l'activation métabolique respiratoire. L'IL-4 
agit aussi sur les granulocytes éosinophiles en augmentant l'expression du CD23 et en inhibant l'expression des récepteurs des $\mathrm{IgG}$.

\section{Interleukine 4 et cellules fibroblastiques et endothéliales}

Des données fragmentaires ont montré que l'IL-4 est capable d'induire la prolifération des fibroblastes et stimule leur production de facteurs de croissance hématopoïétiques comme le GM-CSF. Alors que les interférons bloquent de nombreux effets biologiques de l'IL-4 (en particulier ceux liés au système $\operatorname{IgE}$ ), il a été récemment démontré que l'IL-4 bloque les effets antiviraux des interférons. Fina- lement, les lymphocytes $\mathrm{T}$ adhèrent de façon importante aux cellules endothéliales traitées par l'IL-4 par suite de l'induction de molécules d'adhérence non encore identifiées.

\section{Récepteur de l'interleukine 4}

L'IL-4 se fixe à des récepteurs de haute affinité (de 4 à $12 \times 10^{-11} \mathrm{M}$ ) exprimés en faible nombre (100-1 000) sur pratiquement tous les types cellulaires. L'isolement d'un ADNc codant pour le récepteur de l'IL-4 murine [4-5] a permis le clonage d'un ADNc spécifique du récepteur de l'IL-4 humaine [6, 7]. Ces ADNc codent pour les glycoprotéines matures de 785 et 800 acides aminés (AA) respectivement et de masse moléculaire $130 \mathrm{kDa}$. Elles présentent une homologie significative avec les domaines extracellulaires des récepteurs à l'IL-2 (chaîne p75), IL-3, IL-4, IL-6, IL-7, G-CSF, GMCSF et érythropoiétine. Ces récepteurs, dont la structure moléculaire a été élucidée au cours de l'année 1989, forment donc une nouvelle famille de protéines (figure 3). Deux motifs conservés sont séparés par une séquence de 90-100 AA spécifique de chaque récepteur. Le premier motif, le plus extracellulaire, est composé d'environ $60 \mathrm{AA}$ et contient 4 résidus cystéine conservés. Le second motif, situé près du domaine transmembranaire, est composé d'environ

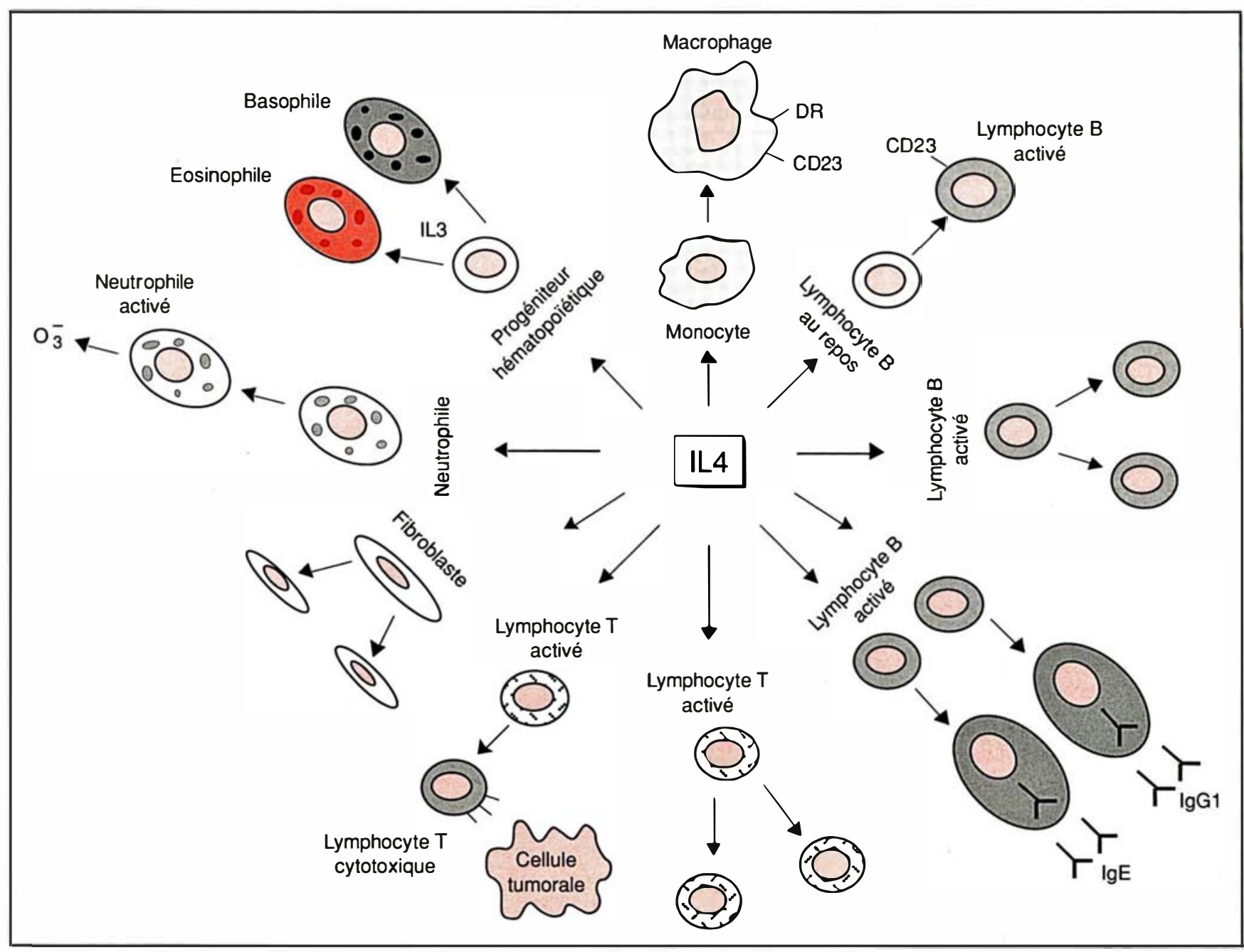

Figure 4. Effets biologiques de l'interleukine 4. 


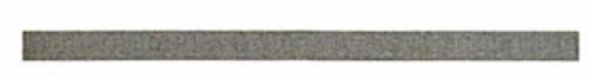

\section{RÉFÉRENCES}

6. Galizzi JP, Zuber CE, Harada N, el al. Molecular cloning of a cDNA encoding the human interleukin-4 receptor. Int Immunol $1990 ; 2: 669-75$.

7. Idzerda RL, March CJ, Mosley B, et al. Human interleukin 4 receptor confers biological responsiveness and defines a novel receptor superfamily. J Exp Med 1990 ; $171: 861-73$

8. Paul WE, Ohara J. B-cell stimulatory factor-1/interleukin 4. Ann Rev Immunol $1987 ; 5: 429-59$.

9. Yokota $\mathrm{T}$, Arai N, de Vries JE, el al. Molecular biology of interleukin 4 and interleukin 5 genes and biology of their products that stimulate $\mathrm{B}$ cells, $\mathrm{T}$ cells and hemopoietic cells. Immunol Rev 1988; 102 : 137-87.

10. Banchereau J. Human interleukin 4 and its receptor. In : Mertelsmann R., Hermann F., eds. Hematopoietic Growth Factors in Clinical Applications. New York : Marcel Dekker, 1990 ; 433-69.

11. Banchereau J. Interleukin 4. In : Thomson AW, ed. Immunology and Molecular Biology of Cytokines. London : Academic Press, 1990 (sous presse).

12. Mosmann TR, Coffman RL. TH and $\mathrm{TH}_{2}$ cells : different patterns of lymphokine secretion lead to different functional properties. Ann Rev Immunol 1989 ; 7 . 145-73.

13. Moore KW, Vieira P, Fiorentino DF, Trounstine ML, Khan TA, Mosmann TR. Homology of cytokine synthesis inhibitory factor (IL-10) to the Epstein-Barr virus gene BCRFI. Science $1990 ; 248$ : 1230-4.

14. Finkelman FD, Holmes J, Katona IM, et al. Lymphokine control on in vivo immunoglobulin isotype selection. Ann Rev Immunol $1990 ; 8$ : 303-33.

15. Tepper RI, Pattengale PK, Leder P. Murine interleukin 4 displays potent antitumor activity in vivo. Cell $1989 ; 57$ :

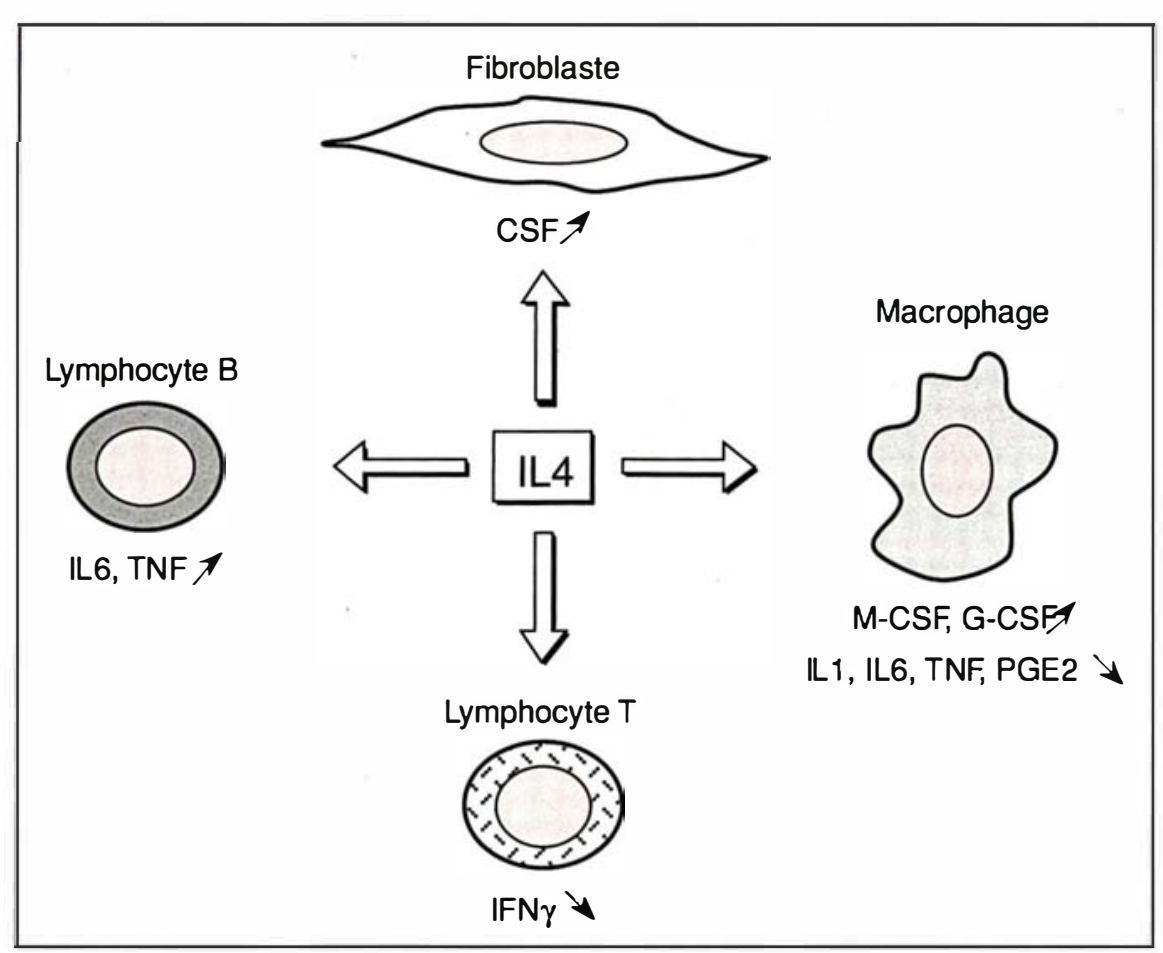

Figure 5. L'interleukine 4 module la production de cytokines de différents types cellulaires. - $\mathbf{A}$ Augmentation ou induction de la sécrétion ; Inhibition ou blocage de la sécrétion. TNF : facteur nécrosant des tumeurs ; IFN $\gamma$ : interféron $\gamma$; G-CSF : facteur de croissance des granulocytes ; M-CSF : facteur de croissance des macrophages et des monocytes; PGE 2 : prostaglandine E2.

$50 \mathrm{AA}$ et comporte un motif conservé Tryp-Ser-X-Tryp-Ser. La longue partie intracytoplasmique contient de nombreux résidus proline et sérine retrouvés dans la chaîne p70 du récepteur à l'IL-2, les récepteurs de l'IL-3 et de l'érythropoïétine. Ces domaines d'homologie pourraient représenter des sites d'interaction avec d'autres protéines constituant ces récepteurs. Dans ce contexte, nos études de pontage de l'IL-4 avec des agents bifonctionnels suggèrent la présence d'un autre composant de 75-80 kDa. Ainsi, comme les récepteurs de l'IL-2, de l'IL-6 et de l'IFN $\gamma$, le récepteur de l'IL-4 pourrait se composer d'au moins deux protéines.

L'activation des lymphocytes $\mathrm{T}$ et $\mathrm{B}$ par différents agents mitogènes induit une augmentation de la densité des récepteurs de l'IL-4 sans affecter ni leur affinité ni leur structure biochimique. L'IL-4 est capable d'augmenter l'expression de son propre récepteur sur les lymphocytes activés.
Comme beaucoup d'autres hormones polypeptidiques, l'IL-4 est internalisée après fixation à son récepteur puis dégradée, vraisemblablement dans les lysosomes.

Les mécanismes intracellulaires mis en jeu par l'IL-4 restent mal définis. Sur les lymphocytes B, l'IL-4 murine ne stimule pas le métabolisme des phospho-inositides, les taux de $\mathrm{Ca}++$ intracellulaires ou la compartimentation de la protéine kinase C. En revanche, elle semble induire la phosphorylation de certaines protéines membranaires. Au contraire, l'IL-4 humaine activerait le métabolisme des phospho-inositides ainsi que l'adénylate cyclase des lymphocytes B normaux.

\section{Conclusions et perspectives}

Il est maintenant clairement établi que l'IL-4 agit sur de nombreux types cellulaires (figure 4, p. 951) et qu'elle peut intervenir à différents 
stades de la différenciation d'une lignée cellulaire particulière. Les effets biologiques de l'IL-4 dépendent de l'environnement cellulaire et des cytokines présentes. De plus, l'IL-4 peut jouer un rôle biologique important de façon indirecte, car elle module la production des cytokines par les cellules $T$, les cellules $B$, les monocytes/macrophages et les fibroblastes (figure 5, p. 952).

Les études in vitro et in vivo ont révélé que l'IL-4 joue un rôle important dans la production d'IgE, mais il reste à démontrer qu'in vivo, on retrouve les effets de l'IL-4 observés sur différentes types cellulaires in vitro. Une étude récente de Tepper et al. [15] a élégamment mis en évidence le rôle antitumoral important de l'IL-4 in vivo. En effet, des lignées de plasmocytomes murins perdent leur caractère tumorigène à la suite de l'introduction d'un gène actif de l'IL-4. L'effet antitumoral serait indirect et se ferait par le recrutement et la stimulation de macrophages et de granulocytes éosinophiles. Ces résultats associés aux propriétés immunostimulatrices de l'IL-4 encouragent certainement à utiliser cette cytokine chez les patients atteints de cancers. A ce niveau, l'IL-4 pourrait faciliter l'élimination des cellules tumorales en augmentant leur expression des molécules d'adhérence LFA-1. En effet, les tumeurs n'exprimant pas le LFA-1 seraient insensibles aux cellules cytotoxiques et échapperaient ainsi aux mécanismes d'immunosurveillance. Finalement, l'IL-4 permet, en combinaison avec l'IL-2, d'engendrer ex vivo des lignées de lymphocytes $\mathrm{T}$ infiltrant les tumeurs (TIL, tumor infiltrating lymphocytes), douées de propriétés cytotoxiques vis-à-vis de la tumeur. Ces cellules, réinjectées in vivo, pourraient s'avérer efficaces pour éliminer les masses tumorales. Les effets inhibiteurs de l'IL-4 sur la production, par les macrophages, des cytokines inflammatoires IL-1, IL-6, et TNF soulèvent la possibilité d'utiliser l'IL-4 comme agent antiinflammatoire, de préférence localement, comme dans le cas de la polyarthrite rhumatoïde.

Les études cliniques fondées sur ces concepts permettront peut-être la mise en évidence d'autres applications thérapeutiques de l'IL-4

\section{Summary}

Interleukin 4

Interleukin 4 (IL-4) is a glycoprotein produced by a restricted number of cells such as $T$ lymphocytes and mast cells. IL-4 acts on numerous cell types including $\mathrm{T}$ and $\mathrm{B}$ lymphocytes, neutrophils, basophils and eosinophils, monocytes/macrophages, fibroblasts and endothelial cells. Its biological effects depend on the status of maturation and activation of a given cell, and on the cellular environment. Moreover, IL-4 can exert biological effects in an indirect fashion as it modulates cytokine production by $\mathrm{T}$ and B lymphocytes, macrophages and fibroblasts. IL-4 plays a major role in $\operatorname{IgE}$ synthesis through the induction of isotype switching in virgin B cells. This property is blocked by interferons. IL- 4 binds to a single class of high affinity receptors which arc expressed at low density on many cell types and composed of at least one glycoprotein of $130 \mathrm{kDa}$. IL-4 may prove useful as an antitumo. ral and anti-inflammatory agent.

\section{TIRÉS A PART}

J. Banchereau.

Avis aux lecteurs : Je serais heureux d'adresser une bibliographie complète sur l'IL-4 aux lecteurs intéressés. 